\title{
DIVERSITY OF DIATOMS IN THE NATURAL, MID-FOREST TEREBOWIEC STREAM - BIESZCZADY NATIONAL PARK
}

\author{
Teresa Noga', Jadwiga Stanek-Tarkowska', Mateusz Rybak', \\ Natalia Kochman-Kędziora ${ }^{2}$, Łukasz Peszek², Anita Pajączek ${ }^{1}$
}

1 Department of Soil Studies, Environmental Chemistry and Hydrology, Faculty of Biology and Agriculture, University of Rzeszów, Zelwerowicza 8B, 35-601 Rzeszów, Poland, e-mail: teresa.noga@interia.pl, jagodastanek@wp.pl, matrybak91@gmail.com, anita.pajaczek@gmail.com

2 Department of Agroecology, Faculty of Biology and Agriculture, University of Rzeszów, Zelwerowicza 8B, 35601 Rzeszów, Poland, e-mail: kochman_natalia@wp.pl, lukaspeszek@gmail.com

Received: 2016.05 .31

Accepted: 2016.08.07

Published: 2016.09 .20

\begin{abstract}
The Terebowiec stream is an $8 \mathrm{~km}$ long watercourse which flows through the strictly protected Bieszczady National Park. The studies on bentic diatoms were conducted between 2013-2015 at three sampling sites along the Terebowiec stream and its unnamed tributary. 260 diatom taxa were identified in the streams, of which 13 taxa were considered as frequent. Chemical analysis showed good and high status of the water. In the studied samples, 27 taxa from the Polish Red List of Algae were determined.
\end{abstract}

Keywords: diatom indices, SPI, GDI, TDI, ecology, taxonomy

\section{INTRODUCTION}

The natural value of the Bieszczady National Park mean it is mentioned in world literature as being among the most interesting European parks. Its attractiveness stems, among others, from the occurrence of numerous herbivorous and predatory mammals, birds of prey, natural ecosystems of Carpathian forests and subalpine meadows with interesting east-Carpathian vegetation. The best known group of plants within the Bieszczady National Park are vascular plants [Zemanek 1991, Winnicki, Zemanek 2009]. Research was also carried out on mosses [Żarnowiec 2010], liverworts [Klama 2013], lichens, fungi and mycetozoa - an overview of the most important literature on these groups of organisms was presented by Winnicki and Zemanek in "Nature in the Bieszczady National Park" [Winnicki, Zemanek 2009].

Algae in the Bieszczady National Park, including diatoms, are very poorly known. Preliminary research on algae was conducted by Wołowski [2011] in the "Wołosate" peat bog (focused mainly on Euglenids) and by ŻelaznaWieczorek [2012] in the sources and upper sec- tions of the River San (diatoms). The first study of diatoms in the River San (below the "Solina" and "Myczkowce" reservoirs) was carried out in connection with the massive growth of Didymosphenia geminata (Lyngbye) M. Schmidt [Kawecka, Sanecki 2003].

In 2010-2011 diatom assemblages in the Wołosaty stream within the San Valley Landscape Park were studied and used to assess water quality via a diatom index [Noga et al. 2014b].

Research has been conducted on diatom assemblages within the catchment area of the Wołosaty stream since 2013 including in the Terebowiec stream. The aim of this study was to investigate diatom diversity in the Terebowiec stream and its tributary.

\section{STUDY AREA}

The Bieszczady National Park is located in the Western Bieszczady, named the "High Bieszczady" by geomorphologists. This area belongs to the Outer Carpathians, built of Carpathian flysch belonging to two structural units - the 
Dukla Nappe and the Silesian Nappe. The rock material from which these formations are built from sedimentary rocks such as sandstones and siltstones, and less commonly marl and conglomerates. Their staggered arrangement in layers of different thicknesses creates the Carpathian flysch. The Bieszczady National Park lies on the border of two climate floors: moderately cold (650 - 1075 m.a.s.1.) and cool (above 1075 m.a.s.1.). The amount of rainfall varies between 1100 and $1200 \mathrm{~mm}$ [Klimaszewski, Starkel 1972, Winnicki, Zemanek 2009].

The Terebowiec stream valley is located in the highest and best-preserved south-eastern part of the Bieszczady National Park, in the Western Bieszczady. The sources of the stream are located on the northern slopes of Tarniczka peak, at an altitude of 1225 m.a.s.l. The length of the stream is $8.01 \mathrm{~km}$. The catchment area is $12.88 \mathrm{~km}^{2}$. The Terebowiec flows into the Wołosatka in Ustrzyki Górne (at an altitude of about 650 m.a.s.1.) and together they form the Wołosaty stream [Czarnecka 2005, Żarnowiec 2010, Klama 2013].

The slopes of the Terebowiec valley are covered in ancient woodland with a dominance of Fagus sylvatica L., a large share of Acer pseu- doplatanus L. and a small amount of Picea abies (L.) H. Karst. Fertile beech forests and acid mountain beech forests are dominant. On the banks of the Terebowiec stream, up to approx. 800 m.a.s.l., patches of mountain alder forests have formed. Above this level there are herb communities of the white butterbur Petasites albus (L.) Gaertn. Headwater areas of the Terebowiec stream and its tributaries are located above the upper limit of the forest among the subalpine meadows. In the valley there are many places where there is outflow of water with characteristic herb vegetation. The Terebowiec stream flows through the strictly protected area, which is one of the best preserved natural parts of the Bieszczady National Park [Przybylska, Kucharzyk 1999, Klama 2013].

\section{METHODS}

Studies were conducted in 2013-2015 on the Terebowiec stream (two sampling sites) and one small, nameless tributary (one sampling site) - Fig. 1 .

Samples were collected in September 2013 only from one, estuary site at Terebowiec stream - sampling site number two. In May and October

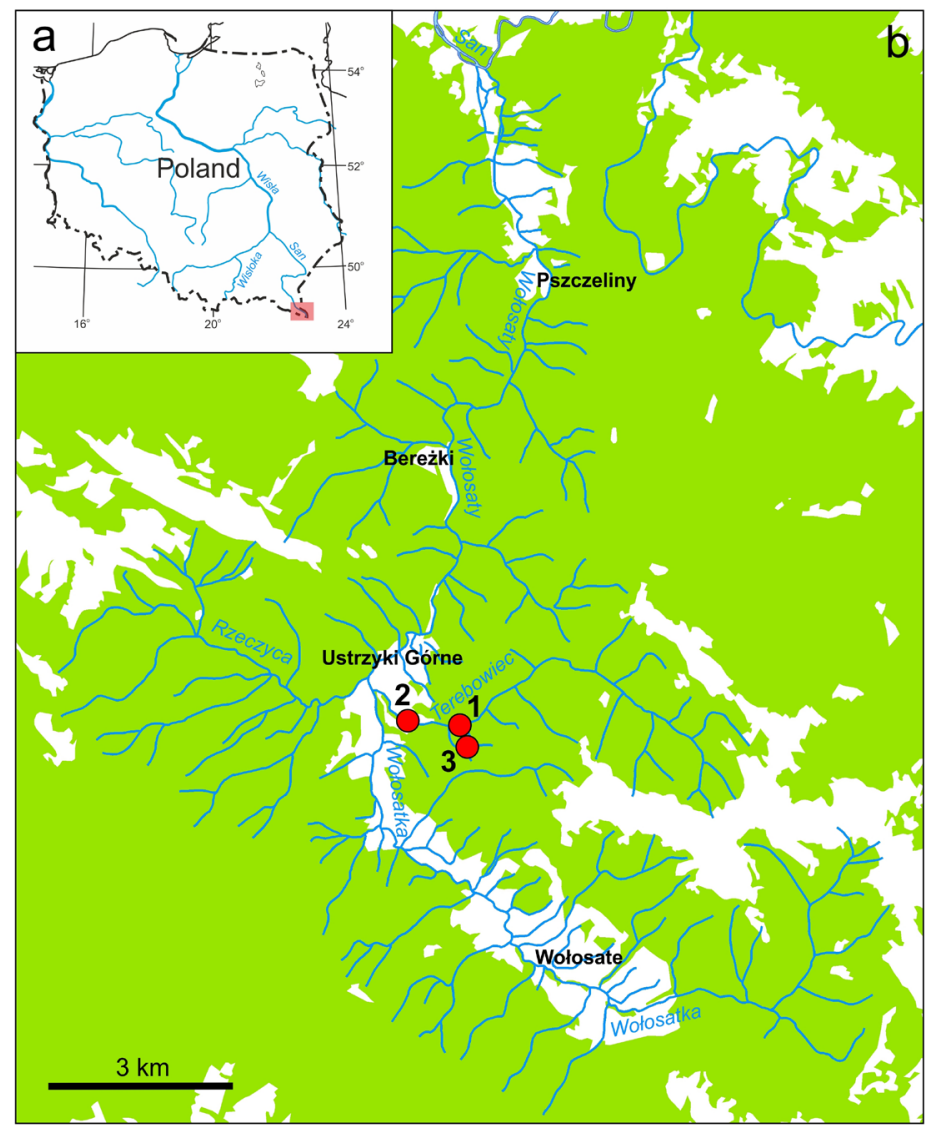

Figure 1. Location of study area (a) and sampling sites (b) on Terebowiec and tributary 
2014 and in October 2015 samples were collected from three sampling sites (two on the Terebowiec stream and one on tributary). Samples were taken from all available habitats, such as stones and aquatic macrophytes (mainly from mosses and algae from the Cladophora genus).

Water $\mathrm{pH}$, electrolytic conductivity, temperature and dissolved oxygen were measured in situ. Water for chemical analysis in the laboratory was taken at the same time. Chemical analyses were performed using a Thermo scientific DIONEX ICS-5000+DC device in the Departmental Laboratory of Analysis of Environmental Health and Materials of Agricultural Origin at the University of Rzeszów.

Collected samples were preserved in a $4 \%$ solution of formalin. Laboratory processing of diatoms was carried out applying methods used by Kawecka [1980] and Noga et al. [2014a]. In order to obtain pure valves of diatoms, part of the obtained material was digested in a chromic acid cleaning mixture and then washed in a centrifuge $(2500 \mathrm{rpm})$. Diatoms were mounted in resin Pleurax (refractive index 1.75).

Diatoms were identified under a Carl Zeiss Axio Imager.A2 light microscope (LM) with a Plan Apochromatic objective $\times 100$ with differentia interference contrast (DIC) for oil immersion. The identification was supported by the following references: Krammer, Lange-Bertalot [1986, 1988, 1991a, b], Lange-Bertalot [2001], Krammer [2000], Levkov [2009], Hofmann et al. [2011]. Selected diatom taxa were presented in the Figure 2.

Species composition of the samples was determined through counting specimens on randomly selected fields of view under light microscope. The number of valves counted was 400 . Species with a content above $5 \%$ in a given assemblage were defined as abundant (Fig. 3).

Analysis of the structure of diatom communities was conducted using OMNIDIA software (version 4.2, database no. 2015a) to determine the ecological status of the water. The software contains an ecological and taxonomic database of diatoms and their bioindication values. The water quality of the stream was assessed based on three diatom indices, for which the range of classes of water quality and the corresponding ecological status was taken according to Żelazowski et al. 2004 and Dumnicka et al. 2006:

\begin{tabular}{|c|l|c|c|c|l|}
\hline $\begin{array}{c}\text { Water } \\
\text { Quality } \\
\text { Class* }\end{array}$ & $\begin{array}{c}\text { Ecological } \\
\text { state }\end{array}$ & IPS & GDI & TDI & $\begin{array}{c}\text { Trophic } \\
\text { state }\end{array}$ \\
\hline I & High & $>17$ & $>17$ & $<35$ & oligotrophic \\
\hline II & Good & $15-17$ & $14-17$ & $35-50$ & $\begin{array}{l}\text { oligo/meso- } \\
\text { trophic }\end{array}$ \\
\hline III & Moderate & $12-15$ & $11-14$ & $50-60$ & mesotrophic \\
\hline IV & Poor & $8-12$ & $8-11$ & $60-75$ & eutrophic \\
\hline V & Bad & $<8$ & $<8$ & $>75$ & $\begin{array}{l}\text { hypertro- } \\
\text { phic }\end{array}$ \\
\hline
\end{tabular}

* According to Regulation of the Minister of the Environment from 22 October 2014 (Dz. U. 2014 No. 0, pos. 1482 .

The SPI (Specific Pollution Sensitivity Index - Coste in CEMAGREF, 1982) and GDI (Generic Diatom Index - Coste \& Aypassorho, 1991) indices are scaled from 1 to 20 (an increase in the value of the indicator means an increase in water quality). The TDI (Trophic Diatom Index, Kelly \& Whitton 1995) is scaled from 1 to 100 (the higher the value, the higher the trophic state of the water). The percentage of pollution-tolerant taxa $(\% \mathrm{PT})$ must be taken into account in the interpretation of the TDI index. There is a possibility of organic pollution if the PT values are above $20 \%$.

According to the Polish Red List of Algae in Poland [Siemińska et al. 2006], the threatened diatoms were attributed to the categories: E - Endangered, V - Vulnerable, R - Rare, and $\mathrm{I}$ - indeterminate.

Statistical analysis with graphical interpretation was made using Canoco software (version 5.03). Taxa with a share in assemblage of more than 5\% from samples collected in 2014 and 2015 were selected for the analysis. To investigate the variation within individual sampling sites and sampling seasons PCA (Principal Component Analysis) was used [Ter Braak, Šmilauer 2012].

\section{RESULTS}

The studied streams were alkaline, most frequently close to a $\mathrm{pH}$ level of 8 . Only in one season, in September 2013, was the $\mathrm{pH}$ level near to circumneutral $(\mathrm{pH}=6.4)$. At all sampling sites the water was highly saturated in oxygen and was characterized by low or average values of electrolytic conductivity $\left(86-246 \mu \mathrm{S} \mathrm{cm}^{-1}\right)$. Also, the values of other studied chemical parameters were repeatedly below the limit of quantification (i.e. 


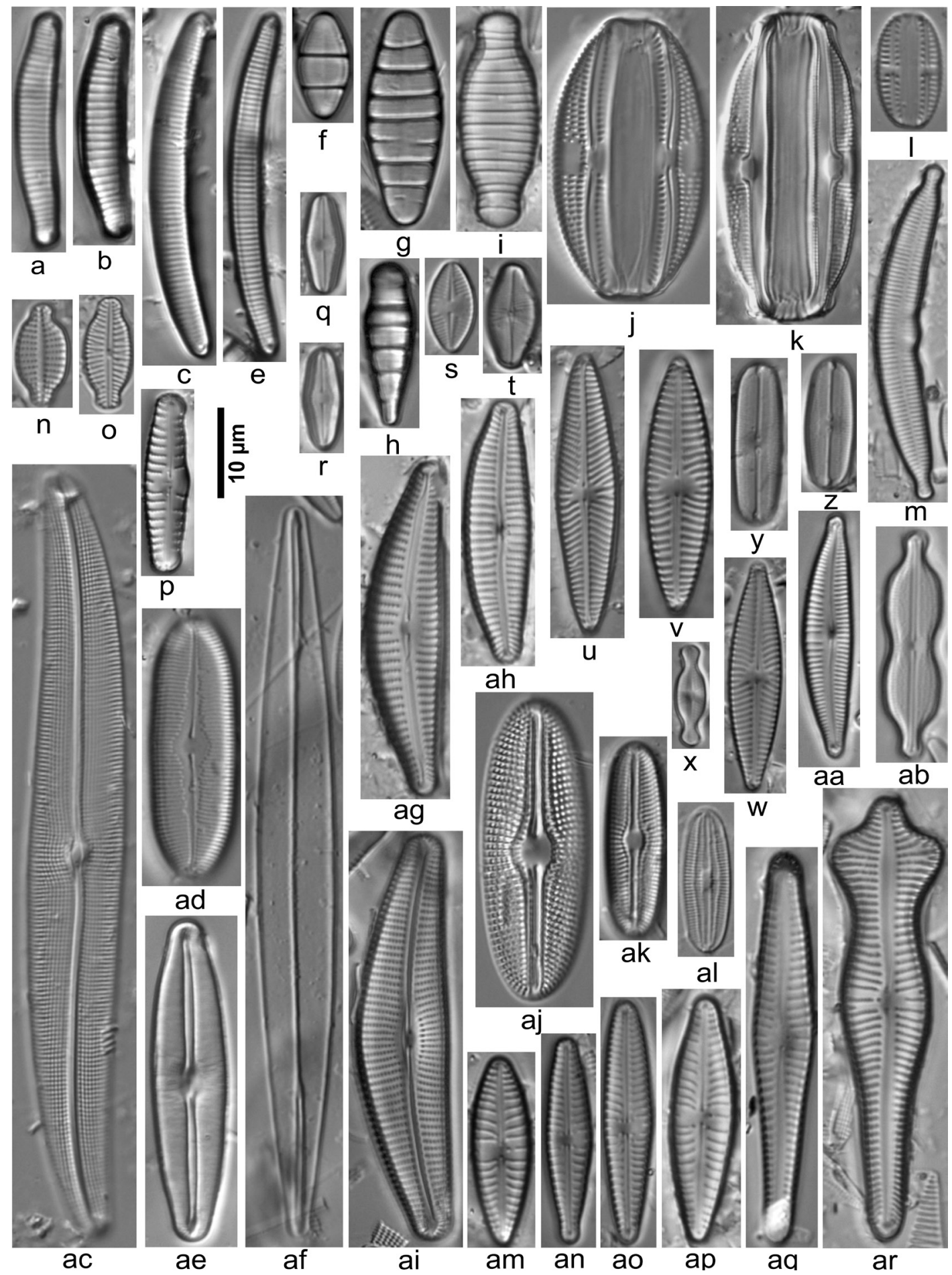

Figure 2. LM micrographs of selected diatom taxa: a-b - Eunotia minor (Kütz.) Grunow, c-e - E. bilunaris (Ehrenb.) Schaarschmidt, f-g - Diatoma mesodon (Ehrenb.) Kütz., h - Meridion circulare var. constrictum (Ralfs) Van Heurck, i - Diatoma ehrenbergii f. capitullata ? (Grunow) Lange-Bert., j - Amphora copulata (Kütz.) Schoeman \& Archibald, k - Halamphora normanii (Rabenh.) Levkov, 1 - Amphora inariensis Krammer, $\mathrm{m}$ - Hannaea arcus (Ehrenb.) Patrick, n-o - Karayevia laterostrata (Hust.) Bukht., p - Reimeria uniseriata Sala, Guerrero \& Ferrario, q-r - Humidophila perpusilla (Grunow) Lowe, Kociolek, Johansen, Van de Vijver, Lange-Bert. \& Kopalová, s - Psammothidium montanum (Krasske) Mayama, $\mathrm{t}$ - Eucocconeis laevis (Østrup) Lange-Bert., u - Navicula oppugnata Hust., v-w - N. antonii Lange-Bert., $\mathrm{x}-N$. medioconvexa Hust., y-z - Fallacia subhamulata (Grunow) D.G. Mann, aa - Delicata delicatula (Kütz.) Krammer, ab - Neidium binodeforme Krammer, ac - Gyrosigma sciotonense (Sulivant) Cleve, ad - Caloneis silicula (Grunow) Cleve, ae Frustulia vulgaris (Thwaites) De Toni, af - Amphipleura pellucida (Kütz.) Kütz., ag - Cymbella hantzschiana Krammer, ah - Cymbella parva (W. Smith) Kirchner, ai - Cymbella compacta Østrup, aj - Diploneis krammeri Lange-Bert. \& Reichardt, ak - D. fontanella Lange-Bert., al - D. petersenii Hust., am, ap - Gomphonema drutelingense Reichardt, an-ao - G. calcifugum Lange-Bert. \& Reichardt, aq - G. subclavatum (Grunow) Grunow, ar - G. acuminatum Ehrenb. 


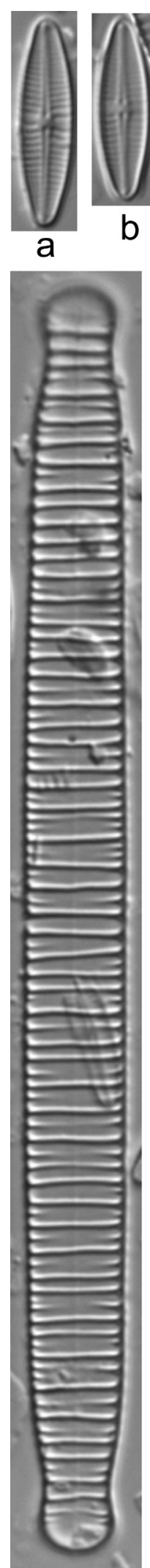

$\mathrm{n}$

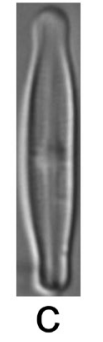

C
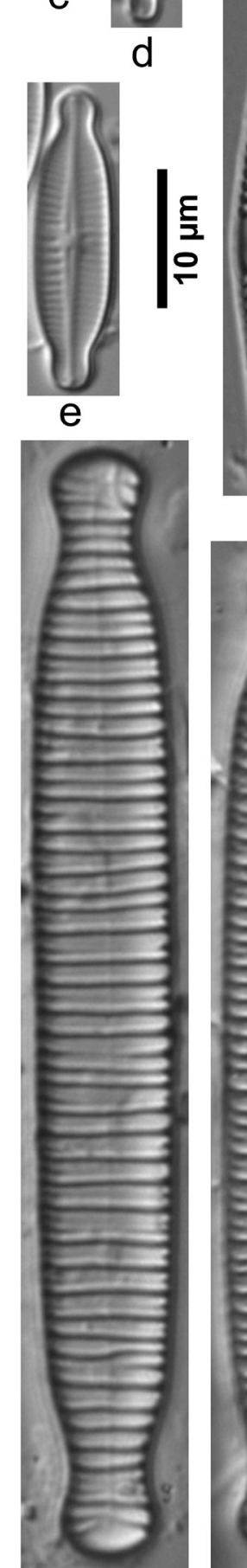

0

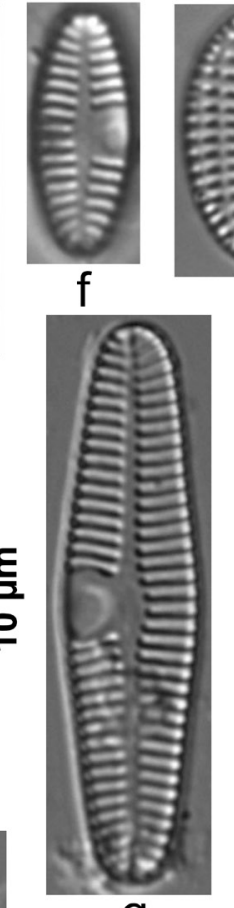

g
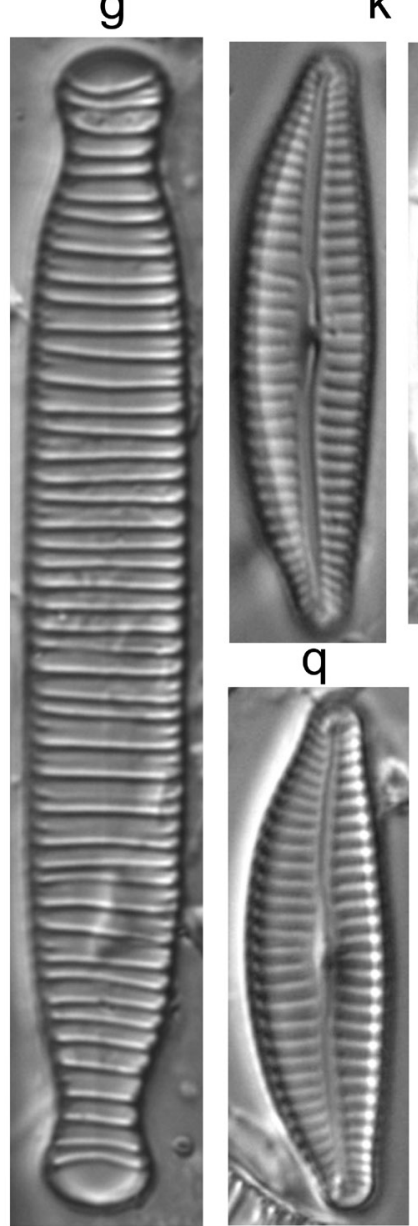

$\mathrm{t}$
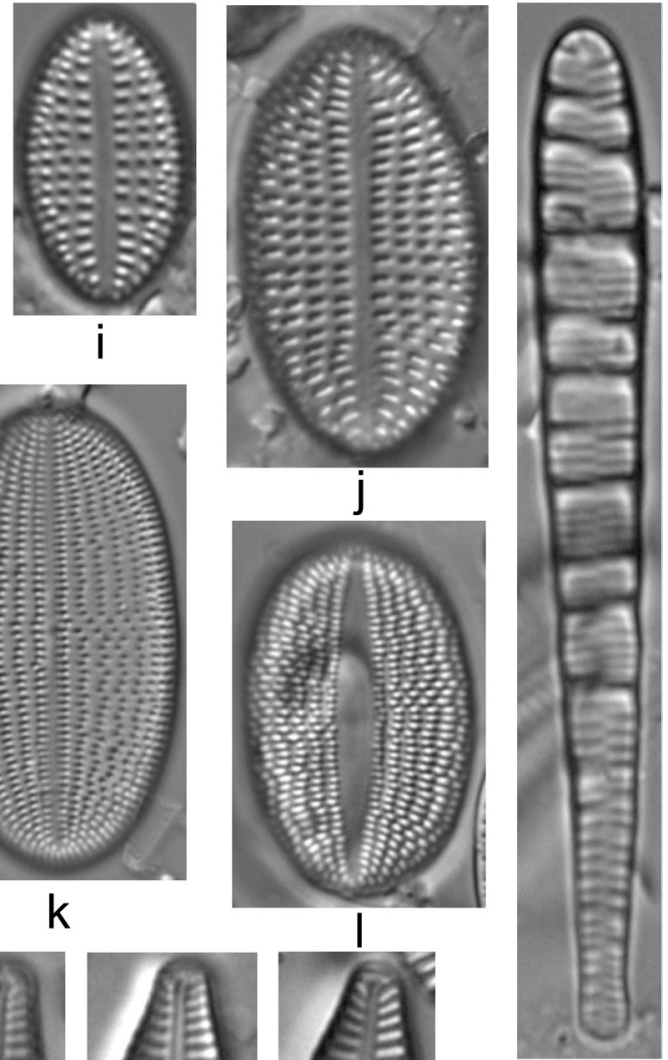

$\mathrm{m}$

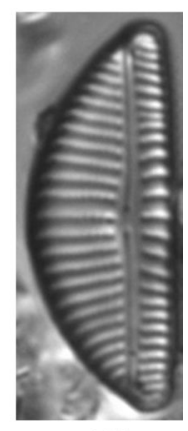

W

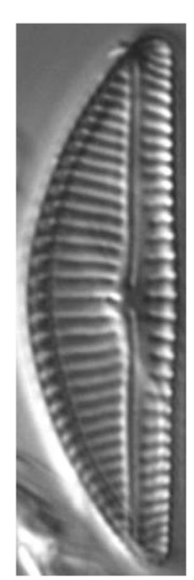

V

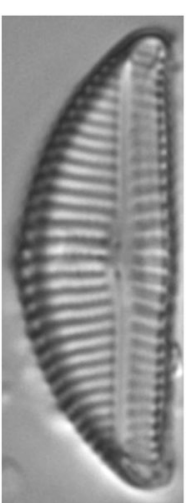

$\mathrm{X}$

Figure 3. LM micrographs of dominant diatom taxa: $\mathrm{a}-\mathrm{b}-$ Achnanthidium pyrenaicum (Hust.) Kobayasi, $\mathrm{c}-\mathrm{d}-A$. minutissimum (Kütz.) Czarnecki var. minutissimum, e - A. thienemannii (Hust.) Lange-Bert., f-g - Planothidium lanceolatum (Brébisson) Round \& Bukht., h-j - Cocconeis pseudolineata (Geitler) Lange-Bert., $\mathrm{k}$ - C. placentula var. lineata (Ehrenb.) Van Heurck, $1-$ C. placentula var. euglypta (Ehrenb.) Grunow, $\mathrm{m}$ - Meridion circulare Agardh var. circulare, n-p - Diatoma ehrenbergii Kütz., q-u - Cymbella parva (W. Smith) Kirchner, v-x - Encyonema silesiacum (Bleisch) D.G. Mann. 
phosphates). Calcium content ranged from 32.41 to $40.30 \mathrm{mg} \mathrm{l}^{-1}$ (Table 1 ).

In studies conducted in 2013-2015, a total of 260 diatom taxa from 57 genera were recorded. The most taxa were recorded from the genera Nitzschia (25), Navicula (23) and Gomphonema (17). Also frequent were Stauroneis (12), Achnanthidium and Pinnularia (11 taxa each). At two sampling sites on the Terebowiec stream 227 diatom taxa were found (123 taxa at site number one and 202 taxa at site number two). On the Terebowiec tributary, 166 taxa were found in total at one sampling site (Table 2). The highest Shannon-Wiener diversity index $\left(\mathrm{H}^{\prime}\right)$ values were recorded on the Terebowiec stream in all sampling seasons (Table 3).

13 diatom taxa were considered as frequent, from which the most (10 taxa) were recorded at site number three, i.e. on the Terebowiec tributary. At all sampling sites Achnanthidium pyrenaicum and Diatoma ehrenbergii f. capitullata were fre- quent species. The most numerous populations were composed of Achnanthidium pyrenaicum, reaching more than a $90 \%$ share in the assemblage at site number one on the Terebowiec stream in Autumn. Diatoma ehrenbergii f. capitullata was the most numerous in the Spring season, reaching more than a $50 \%$ share in the assemblage at site number one. Species from the genera Cocconeis and Planothidium were frequent only on the Terebowiec tributary. In the Spring season on the Terebowiec tributary Diatoma mesodon and Meridion circulare var. circulare, whereas in the Autumn season Achnanthidium minutissimum var. minutissimum were frequent (Fig. 3).

Statistical analysis performed with Canoco software based on the PCA method were separate out sampling site number three, which is located on tributary of the Terebowiec. Differences between sampling seasons are observed only among sampling sites designated on the Terebow-

Tabele 1. The values of physico-chemical parameters measured in Terebowiec stream and tributaries in years 2013-2015.

\begin{tabular}{|c|c|c|c|}
\hline Stream & Terebowiec 1 & Terebowiec 2 & Terebowc tributary \\
\hline Width [m] & $3-7$ & $3-8$ & $0.5-1$ \\
\hline Depth [m] & $0.2-0.4$ & $0.2-0.4$ & około 0.1 \\
\hline Character of bottom & $\begin{array}{c}\text { Stones, larger overgrown by } \\
\text { moss }\end{array}$ & Medium and large stones & $\begin{array}{c}\text { Bottom overgrown by mosses } \\
\text { (to 70\%), between mosses } \\
\text { Cladophora sp. }\end{array}$ \\
\hline Insolation & low & medium & low \\
\hline Temperature $\left[{ }^{\circ} \mathrm{C}\right]$ & $6.1-9.1$ & $8.8-9.3$ & $5.6-9.0$ \\
\hline $\mathrm{pH}$ & $8.2-8.4$ & $6.4-8.4$ & $7.9-8.1$ \\
\hline Conductivity $\left[\mu \mathrm{S} \mathrm{cm}^{-1}\right]$ & $86-138$ & $88-246$ & $106-140$ \\
\hline $\mathrm{O}_{2}\left[\mathrm{mg} \mathrm{l}^{-1}\right]$ & $10.67-10.92$ & $10.77-11.06$ & $10.94-11.02$ \\
\hline $\mathrm{Cl}^{-}\left[\mathrm{mg} \mathrm{l}^{-1}\right]$ & $0.41-1.20$ & $0.87-5.59$ & $0.47-1.03$ \\
\hline $\mathrm{SO}_{4}^{2-}\left[\mathrm{mg} \mathrm{l}^{-1}\right]$ & $12.53-19.63$ & $12.68-27.19$ & 11.95-14.13 \\
\hline $\mathrm{NO}_{3}^{-}\left[\mathrm{mg} \mathrm{l}^{-1}\right]$ & $2.18-2.86$ & $<0,01-2.66$ & $2.89-4.76$ \\
\hline $\mathrm{PO}_{4}{ }^{2-}\left[\mathrm{mg} \mathrm{l}^{-1}\right]$ & $<0,01$ & $<0,01$ & $<0,01$ \\
\hline $\mathrm{NH}_{4}^{+}\left[\mathrm{mg} \mathrm{l}^{-1}\right]$ & $0.06-0.13$ & $<0,01-0.08$ & $0.06-0.09$ \\
\hline $\mathrm{Mg}^{2+}\left[\mathrm{mg} \mathrm{l}^{-1}\right]$ & $5.62-10.10$ & $5.99-8.78$ & $8.32-9.83$ \\
\hline $\mathrm{Ca}^{2+}\left[\mathrm{mg} \mathrm{l}^{-1}\right]$ & $32.41-33.59$ & $32.89-35.31$ & $36.22-40.30$ \\
\hline Dominant taxa & $\begin{array}{l}\text { Achnanthidium } \\
\text { minutissimum } \\
\text { var. minutissimum, } \\
\text { A. pyrenaicum, } \\
\text { Cymbella parva, Diatoma } \\
\text { ehrenbergii } \\
\text { f. capitullata }\end{array}$ & $\begin{array}{l}\text { Achnanthidium pyrenaicum, } \\
\text { A. thienemannii, Cocconeis } \\
\text { placentula } \\
\text { var. euglypta, } \\
\text { C. placentula var. lineata, C. } \\
\text { pseudolineata, Cymbella parva, } \\
\text { Diatoma ehrenbergii } \\
\text { f. capitullata, } \\
\text { Encyonema silesiacum }\end{array}$ & $\begin{array}{l}\text { Achnanthidium minutissimum } \\
\text { var. minutissimum, } \\
\text { A. pyrenaicum, } \\
\text { Cocconeis placentula } \\
\text { var. euglypta, } \\
\text { C. placentula var. lineata, } \\
\text { C. pseudolineata, } \\
\text { Diatoma mesodon, } \\
\text { D. ehrenbergii } \\
\text { f. capitullata, Meridion circulare } \\
\text { var. circulare, Planothidium } \\
\text { lanceolatum, } \\
\text { P. frequentissimum }\end{array}$ \\
\hline
\end{tabular}


Table 2. The list of diatom taxa recorded in Terebowiec stream (sites 1-2) and tributary (site 3) in 2013-2015 ( ${ }^{*}$ category of endangered: E - Endangered, V - Vulnerable, R - Rare, I - Indeterminate).

\begin{tabular}{|c|c|c|c|c|c|c|c|c|c|c|c|}
\hline \multirow{2}{*}{$\begin{array}{c}\text { Date } \\
\text { Number of site }\end{array}$} & \multirow{2}{*}{$\frac{09.13}{2}$} & \multicolumn{3}{|c|}{03.2014} & \multicolumn{3}{|c|}{10.2014} & \multicolumn{3}{|c|}{10.2015} & \multirow{2}{*}{ * } \\
\hline & & 1 & 2 & 3 & 1 & 2 & 3 & 1 & 2 & 3 & \\
\hline Achnanthidium affine (Grunow) Czarnecki & + & & & & + & + & + & + & + & & \\
\hline $\begin{array}{l}\text { Achnanthidium atomus } \\
\text { (Hust.) Monnier, Lange-Bert. \& Ector }\end{array}$ & + & & + & & + & + & & & & & \\
\hline Achnanthidium catenatum (Bily \& Marvan) Lange-Bert. & & & & & & & + & & & & \\
\hline Achnanthidium kranzii (Lange-Bert.) Round \& Bukht. & & & & + & & & & & & + & \\
\hline Achnanthidium lineare W. Smith & + & & & & & + & + & + & + & + & \\
\hline $\begin{array}{l}\text { Achnanthidium minutissimum (Kütz.) Czarnecki } \\
\text { var. minutissimum }\end{array}$ & + & + & + & + & + & + & + & + & & + & \\
\hline Achnanthidium pyrenaicum (Hust.) Kobayasi & + & + & + & & + & + & + & + & + & + & \\
\hline Achnanthidium straubianum (Lange-Bert.) Lange-Bert. & & + & & & & & + & & & & \\
\hline Achnanthidium subatomus (Hust.) Lange-Bert. & & & + & & + & + & + & & & & \\
\hline Achnanthidium thienemannii (Hust.) Lange-Bert. & + & + & + & & + & + & + & + & + & & \\
\hline Achnanthidium cf. pfisterii Lange-Bert. & & & & & & & + & & & & \\
\hline Adlafia minuscula Hust. var. minuscula & & & & + & & & + & + & & & \\
\hline Adlafia cf. suchlandtti (Hust.) Lange-Bert. & & & & & & & + & & & & \\
\hline Amphipleura pellucida (Kütz.) Kütz. & + & + & + & & + & + & + & + & + & + & $\mathrm{R}$ \\
\hline Amphora alpestris Levkov & + & & & & & & & & & & \\
\hline Amphora copulata (Kütz.) Schoeman \& Archibald & & + & + & + & & + & & + & & + & \\
\hline Amphora inariensis Krammer & + & + & + & + & + & + & + & + & + & + & \\
\hline Amphora lange-bertalotii Levkov \& Metzeltin & & & + & & & & & & & & \\
\hline Amphora pediculus (Kütz.) Grunow & + & + & + & + & + & + & + & + & + & + & \\
\hline Aulacoseira granulata (Ehrenb.) Simonsen & & & & & & & & & & + & \\
\hline Aulacoseira sp. & + & & & & & & + & & & & \\
\hline Caloneis aerophila W. Bock & & & & & & + & & & & & \\
\hline Caloneis alpestris (Grunow) Cleve & + & & & & & & & + & + & & \\
\hline Caloneis fontinalis (Grunow) Lange-Bert. \& Reichardt & + & & + & & + & + & + & & + & + & $\mathrm{R}$ \\
\hline Caloneis lancettula (Schulz) Lange-Bert. \& Witkowski & + & + & + & + & & + & & & + & & $\mathrm{R}$ \\
\hline Caloneis schumaniana (Grunow) Cleve & & & + & & & & & & & & $\mathrm{R}$ \\
\hline Caloneis silicula (Grunow) Cleve & & & & + & & & & & & & \\
\hline Caloneis termalis (Grunow) Krammer & & & + & & & & & & & & \\
\hline Campylodiscus hibernicus Ehrenb. & + & & & & & & & & & & \\
\hline Cocconeis disculus (Schumann) Cleve & & & + & & & & & & & & \\
\hline Cocconeis pediculus Ehrenb. & + & + & + & + & + & + & + & + & + & + & \\
\hline Cocconeis placentula var. euglypta (Ehrenb.) Grunow & + & + & + & + & + & + & + & + & + & + & \\
\hline Cocconeis placentula var. lineata (Ehrenb.) Van Heurck & + & + & + & + & + & + & + & + & + & + & \\
\hline Cocconeis placentula Ehrenb. var. placentula & & & + & + & & + & & + & + & + & \\
\hline Cocconeis pseudolineata (Geitler) Lange-Bert. & + & + & + & + & + & + & + & + & + & + & \\
\hline Craticula cuspidata (Kütz.) D.G. Mann & + & & + & & & & & & & & \\
\hline Craticula molestiformis (Hust.) Mayama & & + & & & & & & & & & \\
\hline Cyclostephanos dubius (Fricke) Round & & & + & & & & & & & & \\
\hline Cyclotella meneghiniana Kütz. & & & + & + & & + & & & & + & \\
\hline Cyclotella cf. cyclopuncta Håkansson \& J.R. Carter & & & & & & + & & & & & \\
\hline Cyclotella cf. fottii Hust. & & + & & & & & & & & & \\
\hline Cymatopleura solea (Brébisson) Smith & + & & & & & & & & & & \\
\hline Cymatopleura solea var. apiculata W. Smith & & & & & & & & & + & + & \\
\hline Cymbella compacta Østrup & + & + & + & & + & + & + & + & + & & \\
\hline Cymbella excisa Kütz. & & + & + & & + & & & & + & + & \\
\hline Cymbella hantzschiana Krammer & + & & & & & & & & & & \\
\hline Cymbella helvetica Kütz. & & & + & & & + & + & & & & $\mathrm{R}$ \\
\hline Cymbella hustedtii Krasske & & & & & & & & + & & & \\
\hline Cymbella lanceolata (Ehrenb.) Van Heurck & + & + & + & & & + & & + & + & & \\
\hline Cymbella lange-bertalotii Krammer & & + & & & & & & & & & \\
\hline Cymbella parva (W. Smith) Kirchner & + & + & + & & + & + & + & + & + & + & \\
\hline Cymbopleura amphicephala (Nägeli) Krammer & + & & + & + & & & & & & & \\
\hline Cymbopleura frequens Krammer & & & & & & + & & & & & \\
\hline
\end{tabular}


Table 2 cont.

\begin{tabular}{|c|c|c|c|c|c|c|c|c|c|c|c|}
\hline \multirow{2}{*}{$\begin{array}{c}\text { Date } \\
\text { Number of site }\end{array}$} & \multirow{2}{*}{$\begin{array}{c}09.13 \\
2\end{array}$} & \multicolumn{3}{|c|}{03.2014} & \multicolumn{3}{|c|}{10.2014} & \multicolumn{3}{|c|}{10.2015} & \multirow[t]{2}{*}{ * } \\
\hline & & 1 & 2 & 3 & 1 & 2 & 3 & 1 & 2 & 3 & \\
\hline Cymbopleura naviculiformis (Auerswald) Krammer & & & + & & & & & & & + & \\
\hline Cymbopleura subaequalis (Grunow) Krammer & + & & & & & & & & & & \\
\hline Delicata delicatula (Kütz.) Krammer & & + & + & & & & & & & & \\
\hline Denticula tenuis Kütz. & + & + & + & & + & + & + & + & + & & \\
\hline Diatoma ehrenbergii Kütz. & + & + & + & & + & + & & + & + & & \\
\hline Diatoma ehrenbergii f. capitullata ? (Grunow) Lange-Bert. & + & + & + & & + & + & & + & + & & \\
\hline Diatoma mesodon (Ehrenb.) Kütz. & + & + & + & + & + & + & + & + & + & + & \\
\hline Diatoma moniliformis (Kütz.) D.M. Williams & & & + & & & & & + & + & & \\
\hline Diatoma vulgaris Bory & & + & + & & + & & + & + & + & + & \\
\hline Didymosphenia geminata (Lyngbye) M. Schmidt & + & + & + & & + & + & & + & + & & \\
\hline Diploneis fontanella Lange-Bert. & & & & & & + & & & + & & \\
\hline Diploneis krammeri Lange-Bert. \& Reichardt & & & + & + & & + & + & & + & + & \\
\hline Diploneis petersenii Hust. & & & & + & & & & & & + & \\
\hline Diploneis separanda Lange-Bert. & & + & + & + & + & + & + & & & + & \\
\hline Encyonema auerswaldii Rabenh. & & & & & & + & & & & & \\
\hline Encyonema gauemanii (Meister) Krammer & & & & + & & & & & & & $\mathrm{E}$ \\
\hline Encyonema lange-bertalotii Krammer & + & & + & & + & + & & + & & & \\
\hline Encyonema minutum (Hilse) D.G. Mann & + & + & + & + & + & + & + & + & + & + & \\
\hline Encyonema prostratum (Berkeley) Kütz. & + & + & + & & & + & & & & & \\
\hline Encyonema reichardtii (Krammer) D.G. Mann & & & & & & + & & & + & & \\
\hline Encyonema silesiacum (Bleisch) D.G. Mann & + & + & + & + & + & + & + & + & + & + & \\
\hline Encyonema ventricosum (Agardh) Grunow & + & + & + & & + & + & + & + & + & & \\
\hline Encyonema vulgare Krammer var. vulgare & & + & + & & & + & & + & & & \\
\hline Encyonopsis falaisensis (Grunow) Krammer & & & & & & & & & + & & \\
\hline Encyonopsis krammeri Reichardt & & & & & & & + & & & & \\
\hline Encyonopsis minuta Krammer \& Reichardt & & & + & & & + & & & & & \\
\hline Encyonopsis subminuta Krammer \& Reichardt & & & & & & & + & + & & + & \\
\hline Eolimna minima (Grunow) Lange-Bert. & + & & + & + & + & + & + & + & + & + & \\
\hline Eolimna sp. & & & & & & & + & & & & \\
\hline Epithemia adnata (Kütz.) Brébisson & & & + & & & & & & & & \\
\hline Eucocconeis laevis (Østrup) Lange-Bert. & + & + & + & + & + & + & + & + & + & & \\
\hline Eunotia arcubus Nörpel \& Lange-Bert. & & & & & & + & & & & & \\
\hline Eunotia bilunaris (Ehrenb.) Schaarschmidt & & & + & + & & + & + & & & + & \\
\hline Eunotia exigua (Brébisson) Rabenh. & & & & + & & & & & & & \\
\hline Eunotia fallax Cleve-Euler & & & & & & + & + & & & & \\
\hline Eunotia minor (Kütz.) Grunow & + & & & + & & & & & + & + & \\
\hline Eunotia nymanniana Grunow & & & & & & & + & & & & \\
\hline Eunotia valida Hust. & & & & + & & + & & & & & $\mathrm{I}$ \\
\hline Eunotia cf. novaisiae Lange-Bert. \& Ector & & & & + & & & & & & & \\
\hline Eunotia sp. & & & & + & & & & & & & \\
\hline Fallacia insociabilis (Krasske) D.G. Mann & & & & & & & + & & & & \\
\hline Fallacia lange-bertalotii (Reichardt) Reichardt & & & & & & & + & & & & \\
\hline Fallacia monoculata (Hust.) D.G. Mann & + & & + & & & & + & & & & \\
\hline Fallacia subhamulata (Grunow) D.G. Mann & + & + & & & & + & + & + & + & & $\mathrm{R}$ \\
\hline Fallacia sublucidula (Hust.) D.G. Mann & & & & & & & + & & & & \\
\hline Fallacia tenera (Hust.) D.G. Mann & & + & & & & & & & & & \\
\hline Fallacia sp. & & & & & & & + & & + & & \\
\hline Fragilaria austriaca (Grunow) Lange-Bert. & + & + & + & & & & & + & + & & \\
\hline Fragilaria delicatissima (W. Smith) Lange-Bert. & & + & & & & & & & & & $\mathrm{V}$ \\
\hline Fragilaria gracilis Østrup & + & + & + & & & & + & + & & & \\
\hline Fragilaria pararumpens Lange-Bert., Hofmann \& Werum & + & & & & + & & & & & & \\
\hline Fragilaria perminuta (Grunow) Lange-Bert. & + & + & + & & + & & + & + & + & + & \\
\hline Fragilaria radians (Kütz.) Williams \& Round & & & & & & & & + & & & \\
\hline Fragilaria tenera (W. Smith) Lange-Bert. & & + & & & & & & + & & + & $\mathrm{V}$ \\
\hline Fragilaria vaucheriae (Kütz.) Petersen & + & + & + & & & + & & + & + & & \\
\hline Frustulia vulgaris (Thwaites) De Toni & + & + & + & + & + & + & + & + & + & + & \\
\hline
\end{tabular}


Table 2 cont.

\begin{tabular}{|c|c|c|c|c|c|c|c|c|c|c|c|}
\hline Date & 09.13 & & 20 & & & .201 & & & 20 & & \\
\hline Number of site & 2 & 1 & 2 & 3 & 1 & 2 & 3 & 1 & 2 & 3 & \\
\hline Geissleria acceptata (Hust.) Lange-Bert. \& Metzeltin & + & & + & & + & + & + & + & + & + & $\mathrm{R}$ \\
\hline Geissleria decussis (Østrup) Lange-Bert. \& Metzeltin & + & & & & + & & & + & & & $\mathrm{R}$ \\
\hline Geissleria paludosa (Hust.) Lange-Bert. \& Metzeltin Morphotyp I & & & + & + & & & & & & & \\
\hline Geissleria paludosa (Hust.) Lange-Bert. \& Metzeltin Morphotyp II & & & & & & & & & & + & \\
\hline Gomphonema acuminatum Ehrenb. & + & & & & & & & & + & & \\
\hline Gomphonema calcifugum Lange-Bert. \& Reichardt & + & + & + & + & + & + & & + & + & & \\
\hline Gomphonema clavatum Ehrenb. & & & + & & & + & & & & & \\
\hline Gomphonema drutelingense Reichardt & + & & & + & & & + & & & + & \\
\hline Gomphonema exilissimum (Grunow) Lange-Bert. \& Reichardt & + & & & + & & & + & & & + & \\
\hline Gomphonema micropus Kütz. & & + & + & + & & + & + & + & + & + & \\
\hline Gomphonema minutum (Agardh) Agardh & & & + & & & & & & & & \\
\hline Gomphonema olivaceum (Hornemann) Brébisson var. olivaceum & + & + & + & + & + & + & + & + & + & & \\
\hline Gomphonema parvulum (Kütz.) Kütz. & & & + & + & + & + & & + & & & \\
\hline Gomphonema productum (Grunow) Lange-Bert. \& Reichardt & + & & & + & & & + & & + & + & \\
\hline Gomphonema pumilum (Grunow) Reichardt \& Lange-Bert. & + & + & + & & + & + & + & + & + & + & \\
\hline Gomphonema sarcophagus Gregory & + & & + & & & + & & + & + & & $\mathrm{V}$ \\
\hline Gomphonema subclavatum (Grunow) Grunow & & & + & + & & & + & & + & + & \\
\hline Gomphonema tergestinum (Grunow) Fricke & + & + & + & + & + & + & + & + & + & + & $\mathrm{I}$ \\
\hline Gomphonema utae Lange-Bert. \& Reichardt & + & & & & & & & & & & \\
\hline Gomphonema cf. angustatum (Kütz.) Rabenh. & & & & & & & + & & & & \\
\hline Gomphonema sp. & & + & + & & & + & + & & & & \\
\hline Gyrosigma attenuatum (Kütz.) Rabenh. & + & + & & & & & & & & & \\
\hline Gyrosigma obtusatum (Sulivant \& Wormley) Boyer & + & & & & & & & & & & \\
\hline Gyrosigma sciotonense (Sulivant) Cleve & + & & + & & & + & & & + & & \\
\hline Halamphora montana (Krasske) Levkov & + & & & + & & & & & + & & \\
\hline Halamphora normanii (Rabenh.) Levkov & + & & & + & & & + & & + & + & \\
\hline Halamphora normanii var. undulata (Krasske) Levkov & + & & & & & & & & & & \\
\hline Hannaea arcus (Ehrenb.) Patrick & + & + & + & + & + & + & + & + & + & & \\
\hline Hantzschia abundans Lange-Bert. & & & + & & & & & & & & \\
\hline Hantzschia amphioxys (Ehrenb.) Grunow & & & + & + & & & & & & + & \\
\hline Hantzschia calcifuga Reichardt \& Lange-Bert. & & & + & & & + & + & & & & \\
\hline $\begin{array}{l}\text { Humidophila brakkaensis (Petersen) Lowe, Kociolek, } \\
\text { Johansen, Van de Vijver, Lange-Bert. \& Kopalová }\end{array}$ & & & + & & & & + & & + & + & \\
\hline $\begin{array}{l}\text { Humidophila contenta (Grunow) Lowe, Kociolek, } \\
\text { Johansen, Van de Vijver, Lange-Bert. \& Kopalová }\end{array}$ & & & + & + & & + & & + & + & & \\
\hline $\begin{array}{l}\text { Humidophila perpusilla (Grunow) Lowe, Kociolek, } \\
\text { Johansen, Van de Vijver, Lange-Bert. \& Kopalová }\end{array}$ & + & + & + & + & + & + & + & + & + & + & \\
\hline Humidophila sp. & & + & + & & + & + & & & & & \\
\hline Karayevia laterostrata (Hust.) Bukht. & + & & & + & & + & & & & + & \\
\hline Luticola frequentissima Levkov, Metzeltin \& Pavlov & & & + & & + & & & & & & \\
\hline Luticola mutica (Kütz.) D.G. Mann & + & + & + & & & + & & & + & & \\
\hline Luticola cf. acidoclinata Lange-Bert. & & & & + & & + & & & + & & \\
\hline Mayamaea atomus (Kütz.) Grunow var. atomus & & & & & & & & & & + & \\
\hline Mayamaea atomus var. permitis (Hust.) Lange-Bert. & & & & & & & + & & & & \\
\hline Melosira varians Agardh & & & + & & & & & & & & \\
\hline Meridion circulare Agardh var. circulare & + & + & + & + & + & + & + & + & + & + & \\
\hline Meridion circulare var. constrictum (Ralfs) Van Heurck & + & & + & + & & & & & & & \\
\hline Navicula antonii Lange-Bert. & & + & + & + & & + & + & + & + & & \\
\hline Navicula associata Lange-Bert. & + & & & & & & & & & & \\
\hline Navicula capitatoradiata Germain & & & & & & & & & + & & \\
\hline Navicula cryptocephala Kütz. & + & & + & + & + & + & & + & + & & \\
\hline Navicula cryptofallax Lange-Bert. \& Hofmann & + & & & & & & & & & & \\
\hline Navicula cryptotenella Lange-Bert. & + & + & + & + & + & + & & + & + & & \\
\hline Navicula cryptotenelloides Lange-Bert. & + & + & + & & + & + & + & + & + & & \\
\hline Navicula gregaria Donkin & & & + & + & & & + & + & + & + & \\
\hline Navicula lanceolata (Agardh) Ehrenb. & & + & + & + & & & & & & & \\
\hline Navicula lundii Reichardt & & & & + & & & + & & & + & \\
\hline Navicula medioconvexa Hust. & & & & & & + & & & & + & \\
\hline
\end{tabular}


Table 2 cont.

\begin{tabular}{|c|c|c|c|c|c|c|c|c|c|c|c|}
\hline \multirow{2}{*}{$\begin{array}{c}\text { Date } \\
\text { Number of site }\end{array}$} & \multirow{2}{*}{$\begin{array}{c}09.13 \\
2\end{array}$} & \multicolumn{3}{|c|}{03.2014} & \multicolumn{3}{|c|}{10.2014} & \multicolumn{3}{|c|}{10.2015} & \multirow{2}{*}{ * } \\
\hline & & 1 & 2 & 3 & 1 & 2 & 3 & 1 & 2 & 3 & \\
\hline Navicula moskalii Metzeltin, Witkowski \& Lange-Bert. & & & & & & + & & & & & $\mathrm{R}$ \\
\hline Navicula novaesiberica Lange-Bert. & + & & & & & & & & & & \\
\hline Navicula oppugnata Hust. & + & & & & + & + & + & & + & & $\mathrm{R}$ \\
\hline Navicula radiosa Kütz. & + & + & + & & + & + & + & + & + & + & \\
\hline Navicula recens (Lange-Bert.) Lange-Bert. & + & + & & & + & + & & & + & + & \\
\hline Navicula reinhardtii (Grunow) Grunow & & & & & & & & & + & & \\
\hline Navicula splendicula Van Landingham & + & + & + & + & + & + & + & + & + & & $\mathrm{V}$ \\
\hline Navicula tenelloides Hust. & + & & & + & & + & + & & & + & \\
\hline Navicula tripunctata (O.F. Müller) Bory & + & + & + & + & + & + & + & + & + & & \\
\hline Navicula cf. difficillima Hust. & & & & & & & & & & + & \\
\hline Navicula cf. exilis Grunow & & & & & & & + & & & & \\
\hline Navicula cf. notha Wallace & & & & & & & + & & & & \\
\hline Neidium alpinum Hust. & & & + & + & & & + & & + & & $\mathrm{E}$ \\
\hline Neidium binodeforme Krammer & + & & & + & & & & + & + & & \\
\hline Nitzschia acicularis (Kütz.) W. Smith & + & & & & & & & & & & \\
\hline Nitzschia acidoclinata Lange-Bert. & + & & + & + & + & + & + & & + & + & \\
\hline Nitzschia adamata Hust. & & + & & & & & & & & & \\
\hline Nitzschia alpina Hust. & & & & & & + & & & & & \\
\hline Nitzschia amphibia Grunow & + & & & & & + & & & & & \\
\hline Nitzschia dissipata (Kütz.) Grunow ssp. dissipata & + & & + & + & + & + & & + & + & + & \\
\hline Nitzschia dissipata var. media (Hantzsch) Grunow & & & & & & & & & & + & \\
\hline Nitzschia draveliensis Coste \& Ricard & + & & & & & & & & & & \\
\hline Nitzschia fonticola Grunow & + & + & + & & + & + & + & + & + & & \\
\hline Nitzschia frustulum (Kütz.) Grunow & + & & + & & + & + & & & + & & \\
\hline Nitzschia hantzschiana Rabenh. & & & + & & & & & & + & & \\
\hline Nitzschia heufleriana Grunow & + & + & + & & + & + & & + & + & & \\
\hline Nitzschia intermedia Hantzsch & & + & + & & & & & & & & \\
\hline Nitzschia linearis (Agardh) W. Smith & + & & + & + & + & + & + & + & + & + & \\
\hline Nitzschia palea (Kützing) W. Smith & + & & & & & & & & & + & \\
\hline Nitzschia paleacea (Grunow) Grunow & & & + & & & & & & & & \\
\hline Nitzschia perminuta (Grunow) Peragallo & & + & & & & & & & & & \\
\hline Nitzschia pura Hust. & & & + & & & & & & & & \\
\hline Nitzschia pusilla Grunow & + & & & + & & & & & & + & \\
\hline Nitzschia recta Hantzsch & + & & & + & + & + & + & + & + & & \\
\hline Nitzschia sigma (Kütz.) W. Smith & & & + & & & & & & + & & \\
\hline Nitzschia sigmoidea (Nitzsch) W. Smith & + & & + & & & & & & + & & \\
\hline Nitzschia solgensis Cleve-Euler & + & & & & & & & & & & \\
\hline Nitzschia sublinearis Hust. & + & + & + & & + & & + & & & & \\
\hline Nitzschia tenuis W. Smith & + & & & + & & + & & & & + & \\
\hline Pinnularia appendiculata (Agardh) Schaarschmidt & & & & & & & + & & & & \\
\hline Pinnularia borealis Ehrenb. var. borealis & & & + & + & & + & & & + & & \\
\hline Pinnularia brebissonii (Kützing) Rabenh. & & & & & & & & & + & & \\
\hline Pinnularia isselana Krammer & + & + & + & & & & + & & + & & \\
\hline Pinnularia marchica Schönfelder & & & & + & & + & & & & & \\
\hline Pinnularia obscura Krasske & & & + & + & & & & & & + & \\
\hline Pinnularia schoenfelderi Krammer & + & & + & + & & + & & & & & $\mathrm{E}$ \\
\hline Pinnularia sinistra Krammer & + & & & & & & & & & & \\
\hline Pinnularia subgibba var. undulata Krammer & & & & + & & & & & & & \\
\hline Pinnularia subrupestris Krammer & + & & & + & & & & & + & & $\mathrm{E}$ \\
\hline Pinnularia silvatica Petersen & & & & + & & & + & & & + & \\
\hline Placoneis ignorata (Schimanski) Lange-Bert. & & & + & & & & & & & & \\
\hline Placoneis paraelginensis Lange-Bert. & + & & & + & & + & + & & & + & \\
\hline Planothidium dubium (Grunow) Round \& Bukht. & & & & & & & & & & + & \\
\hline Planothidium ellipticum (Cleve) Round \& Bukht. & & & + & & & & & & & & \\
\hline Planothidium frequentissimum (Lange-Bert.) Round \& Bukht. & + & + & + & + & & + & + & + & + & + & \\
\hline Planothidium lanceolatum (Brébisson) Round \& Bukht. & + & + & + & + & & + & + & + & + & + & \\
\hline
\end{tabular}


Table 2 cont.

\begin{tabular}{|c|c|c|c|c|c|c|c|c|c|c|c|}
\hline \multirow{2}{*}{$\begin{array}{c}\text { Date } \\
\text { Number of site }\end{array}$} & \multirow{2}{*}{$\frac{09.13}{2}$} & \multicolumn{3}{|c|}{03.2014} & \multicolumn{3}{|c|}{10.2014} & \multicolumn{3}{|c|}{10.2015} & \multirow{2}{*}{ * } \\
\hline & & 1 & 2 & 3 & 1 & 2 & 3 & 1 & 2 & 3 & \\
\hline Planothidium biporomum (Hohn \& Hellerman) Lange-Bert. & & & + & + & & + & + & & + & + & \\
\hline Psammothidium bioretii (Germain) Bukht. \& Round & & & & + & & & & & & & \\
\hline Psammothidium daonense (Lange-Bert.) Lange-Bert. & & + & + & + & & + & & + & + & + & \\
\hline Psammothidium grischunum (Wuthrich) Bukht. \& Round & + & + & + & + & + & + & + & + & + & + & \\
\hline Psammothidium lauenburgianum (Hust.) Bukht. \& Round & + & & & + & & & + & & + & + & $\mathrm{V}$ \\
\hline Psammothidium montanum (Krasske) Mayama & & & + & & & & + & + & + & + & \\
\hline Psammothidium subatomoides (Hust.) Bukht. \& Round & & + & + & & & & & & & & $\mathrm{V}$ \\
\hline Reimeria ovata (Hust.) Levkov \& Ector & & + & + & + & + & + & & + & + & & \\
\hline Reimeria sinuata (Gregory) Kociolek \& Stoermer & + & + & + & + & + & + & + & + & + & + & \\
\hline Reimeria uniseriata Sala, Guerrero \& Ferrario & + & + & + & & + & + & + & & + & & \\
\hline Rhoicosphenia abbreviata (Agardh) Lange-Bert. & + & + & + & + & + & + & + & + & + & & \\
\hline Sellaphora joubaudii (Germain) Aboal & & & & & & + & & & & + & \\
\hline Sellaphora laevissima (Kütz.) D.G. Mann & & & + & & & & & & & & \\
\hline Sellaphora nana (Hust.) Lange-Bert., Cavacini, Tagliaventi \& Alfinito & & & + & & & & & & & & \\
\hline Sellaphora pseudopupula (Krasske) Lange-Bert. & & & & + & + & + & + & & + & & $E$ \\
\hline Sellaphora pupula (Kütz.) Mereschkovsky & + & & & & & & & + & + & + & \\
\hline Sellaphora seminulum (Grunow) D.G. Mann & + & & + & & & + & + & & + & + & \\
\hline Sellaphora stroemii (Hust.) D.G. Mann & & & + & & & + & & & + & & \\
\hline Simonsenia delognei (Grunow) Lange-Bert. & & & & & & & + & & & & \\
\hline Stauroneis amphicephala Kütz. & & + & & & & & & & & & \\
\hline Stauroneis anceps Ehrenb. & & & + & + & & + & + & & & + & \\
\hline Stauroneis gracilis Ehrenb. & & & + & + & & & & & & + & $\mathrm{V}$ \\
\hline Stauroneis intricans Van de Vijver \& Lange-Bert. & & & & & + & + & & & + & & \\
\hline Stauroneis kriegerii Patrick & & & + & + & & & & & + & + & \\
\hline Stauroneis lauenburgiana Hust. & & & & + & & & & & & + & \\
\hline Stauroneis parathermicola Lange-Bert. & & & & + & & + & + & & & & \\
\hline Stauroneis phoenicenteron (Nitzsch) Ehrenb. & & & & + & & & & & & & $\mathrm{V}$ \\
\hline Stauroneis pseudoagrestis Lange-Bert. \& Werum & + & & & + & & & & & & & \\
\hline Stauroneis separanda Lange-Bert. \& Werum & & & & & & & & + & + & & \\
\hline Stauroneis smithi Grunow & + & & & + & & & & & & + & \\
\hline Stauroneis thermicola (Petersen) Lund & + & & & + & & & + & & + & + & $\mathrm{R}$ \\
\hline Staurosira venter (Ehrenb.) Grunow & + & & & & & & & & & & \\
\hline Staurosirella pinnata (Ehrenb.) Williams \& Round & + & & & & & + & & & & & \\
\hline Stephanodiscus sp. & & & + & & & & & & & & \\
\hline Surirella angusta Kütz. & & + & + & + & + & + & + & & + & + & \\
\hline Surirella minuta Brébisson & & + & + & + & & & & & + & + & \\
\hline Surirella terricola Lange-Bert. \& Alles & + & + & & + & & + & & & & & \\
\hline Tabellaria flocculosa (Roth) Kütz. & & & + & & & & & & & & \\
\hline Ulnaria acus (Kütz.) Lange-Bert. & & + & + & + & + & + & + & + & + & & \\
\hline Ulnaria ulna (Nitzsch) Compère & + & & + & & & + & + & + & + & + & \\
\hline
\end{tabular}

Table 3. The values of diatom indices SPI, GDI, TDI, \%PT and Shannon-Wiener diversity index (H') calculated for individual sites in the Terebowiec stream (sites 1-2) and tributary (site 3) in 2013-2015.

\begin{tabular}{|c|c|c|c|c|c|c|c|c|c|c|}
\hline Date & 09.2013 & \multicolumn{3}{|c|}{03.2014} & \multicolumn{3}{|c|}{10.2014} & \multicolumn{3}{|c|}{10.2015} \\
\hline Site & 2 & 1 & 2 & 3 & 1 & 2 & 3 & 1 & 2 & 3 \\
\hline IPS & 18.2 & 16.1 & 16.6 & 19.1 & 19.0 & 18.7 & 16.8 & 19.1 & 19.1 & 16.0 \\
\hline GDI & 16.0 & 15.1 & 15.2 & 16.6 & 16.8 & 16.8 & 13.7 & 17.1 & 16.9 & 14.4 \\
\hline TDI & 60.7 & 41.2 & 55.3 & 26.3 & 70.2 & 68.7 & 52.2 & 68.2 & 69.4 & 49.8 \\
\hline \%PT & 4.7 & 2.5 & 4.8 & 3.1 & 1.6 & 2.4 & 2.9 & 1.9 & 1.1 & 5.2 \\
\hline $\mathrm{H}^{\prime}$ & 3.89 & 3.52 & 4.24 & 4.74 & 2.11 & 2.52 & 4.78 & 2.58 & 2.21 & 4.89 \\
\hline \multirow{2}{*}{\multicolumn{2}{|c|}{$\begin{array}{c}\text { Ecological status } \\
\text { IPS, GDI, TDI }\end{array}$}} & \multirow{2}{*}{\multicolumn{2}{|c|}{ high }} & \multicolumn{2}{|c|}{ good } & \multicolumn{2}{|c|}{ moderate } & poor & \multicolumn{2}{|c|}{ bad } \\
\hline & & & & & & & & & & \\
\hline
\end{tabular}


iec stream - two groups from Spring and Autumn were clearly separated (Fig. 4).

The SPI and GDI indices values showed good or high ecological status in all sampling seasons, corresponding with a II or I water quality class. The TDI trophic index had the highest values (26.3-70.2), indicating a usually poor or moderate ecological status, especially in Autumn periods. Only on the Terebowiec tributary and in Spring on the Terebowiec did the TDI values indicate a good or moderate ecological status. \%PT values were low and ranged from 1.1 to $5.2 \%$ (Table 3 ).

In the studied samples, 27 diatom taxa were recorded from the Polish Red List of Algae [Siemińska et al. 2006]. This represents over $10 \%$ of the total number of identified taxa. Five taxa were found from the E category - endangered: Encyonema gauemanii (Meister) Krammer, Neidium alpinum Hust., Pinnularia schoenfelderi Krammer, P. subrupestris Krammer and Sellaphora pseudopupula (Krasske) Lange-Bert. Many rare diatom taxa were found from the $\mathrm{Na}$ vicula genus: Navicula medioconvexa Hust., $\mathrm{Na}$ vicula moskalii Metzeltin, Witkowski \& LangeBert., Navicula oppugnata Hust. and Navicula splendicula Van Landingham.

\section{DISCUSSION}

Waters of streams and rivers of the upper San basin are relatively clean. However, a large influx of tourists during the summer months in the Bieszczady area means streams have an increased concentration of nitrogen and phosphates. Water pollution is causing adverse changes in the streams of the most value, and most at risk include the Wołosatka, Rzeczyca and Terebowiec [Kukuła 2002]. During the tourist season (mainly in May, July, August) in Wołosate village there are elevated levels of phosphate, ammonium and biochemical oxygen demand (BOD), primarily in the Wołosatka stream, but also to a lesser extent in the Wołosaty, Dębowiec and Rzeczyca streams [Kukuła, Szczęsny 2000]. In 2009-2010 research on benthic macrofauna was conducted on the Wołosatka, Wołosaty, Rzeczyca and Terebowiec streams.

The physicochemical parameters of water also showed elevated values of phosphorus, ammonium, BOD (especially in the summer months), and as such classify the water below a II class of chemical status [Galas et al. 2014].

Most of the physicochemical parameters in the waters of the Terebowiec stream and its tribu-

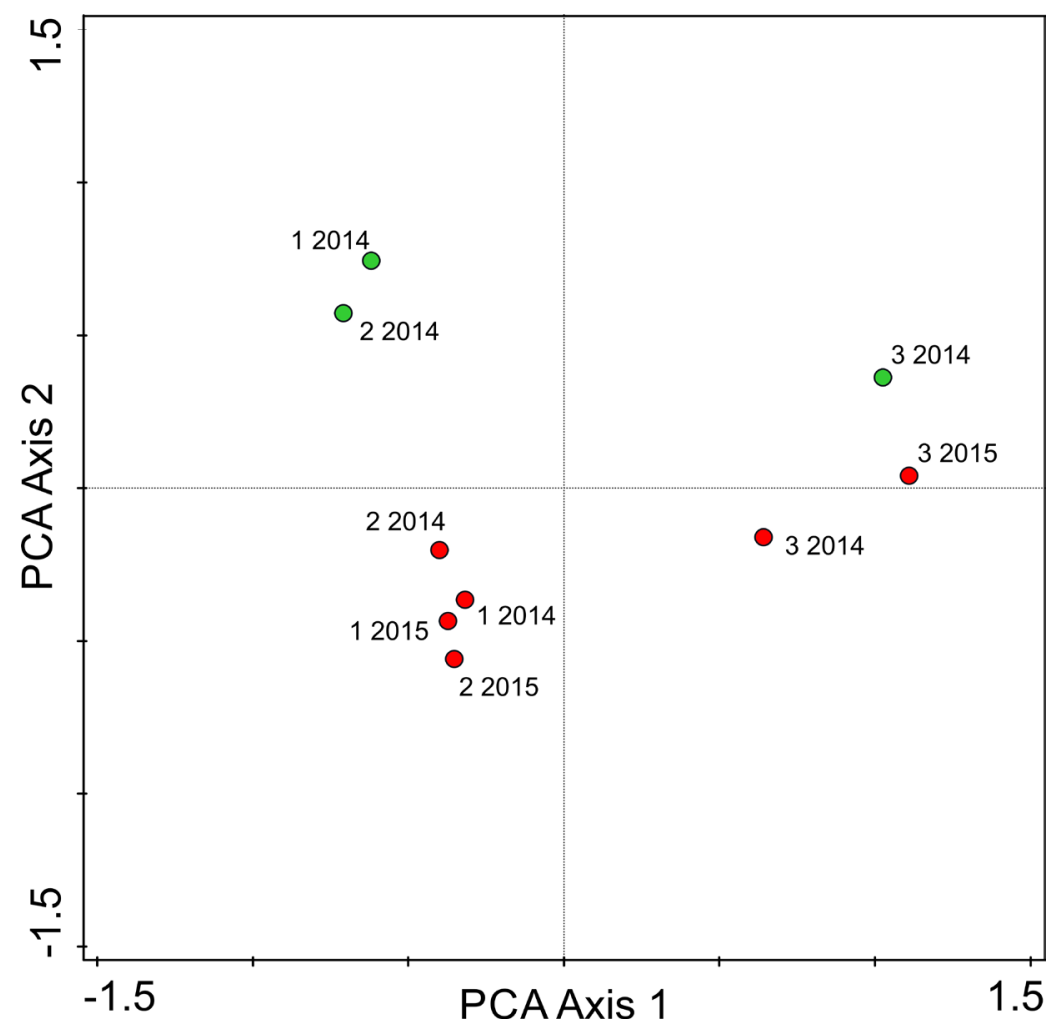

Figure 4. PCA ordination of sampling sites determined by relative community composition.

Clusters represent sites of similarity based on the sampling season (green points - sampling sites form Spring, red points - sampling sites from Autumn). 
tary in the years 2013-2015 were characteristic for the I class of water quality. Only the nitrate content was elevated and corresponded to a II class of water quality. Much lower values of nitrate were measured in the River San during studies in 2010-2011 in the area of the San Valley Landscape Park. All the investigated chemical parameters qualified waters of the River San as I class of water quality [Noga et a. 2014b]. The upper part of the San brings much more water compared to smaller and shallow streams, as with the Terebowiec, so that even during the summer at low water levels it has better water quality. The Terebowiec stream, like most rivers in southeastern Poland, is characterized by high species richness of diatoms. Greater species richness was observed on the River San in the area of the San Valley Landscape Park [Noga et al. 2014b] and in springs and source sections of the upper San tributaries [Żelazna-Wieczorek 2012]. Many rivers and streams in Podkarpacie state have a similar or higher species richness of diatoms, but in many cases the studies have been conducted on a larger number of sampling sites [Noga 2012, Pajączek et al. 2012, Noga et al. 2013a, b, 2014a, Peszek et al. 2015].

The main dominant species in each period of study at both studied sites in the Terebowiec stream was Achnanthidium pyrenaicum (Hust.) Kobayasi. In Autumn, the number of the species often exceeds $90 \%$ participation in the assemblage. It is a species that has optimum occurrence in oligo- and mesotrophic, calcium-rich waters flowing in mountainous regions and highlands [Hofmann et al. 2011]. According to Hofmann [1994] it is a tolerant species, which prefers alkaline water with a $\mathrm{pH}>7$. In south-eastern Poland it develops in large numbers in many rivers and streams, as one of the most common diatoms [Noga 2012; Noga et al. 2013b; 2014a, b]. Research conducted by Potapowa [2006] in rivers and streams in north-western Russia showed that A. pyrenaicum also frequently develops there and is often the dominant species. Similarly, other species develop numerously - Diatoma ehrenbergii Kütz. has an optimum occurrence in the mountain standing and flowing waters, rich in calcium, wherein it often develops massively [Hofmann et al. 2011; Bąk et al. 2012]. At the sites in the Terebowiec stream, D. ehrenbergii f. capitulata (Grunow) Lange-Bert. developed in the spring season in large numbers. It seems that it has a similar occurrence and ecology to D. ehren- bergii because they co-occur together, although D. ehrenbergii f. capitulata created much more numerous populations. In the tributary of the Terebowiec numerously developed species were from the genera Cocconeis (C. placentula var. euglypta, C. placentula var. lineata and C. pseudolineata) and Planothidium (P. frequentissimum and $P$. lanceolatum). All varieties of $C$. placentula are epiphitic taxa and in some places develop massively, however environmental requirements and the presence of $C$. pseudolineta are inaccurately understood, probably it prefers water with an average or higher content of electrolytes and trophy. Because the Terebowiec tributary was numerously developed by green algae, mainly from the genus Cladophora and in places by mosses, so epiphitic diatom species found here favorable conditions for growing. Both species of the genus Planothidium have broad ecological amplitude and develop with great stability in all types of flowing water [Hofmann et al. 2011, Bąk et al. 2012]. In the spring numerous populations of $\mathrm{Me}$ ridion circulare var. circulare were observed, a species which also develops with high stability in various types of water, as well as Diatoma meso$d o n$ - a species frequently observed in low-electrolyte and oligo-mesotrophic waters [Hofmann et al. 2011, Bąk et al. 2012]. In Podkarpacie these species are common, but many populations are very rarely observed, usually in the upper reaches of streams and mainly in the spring season [Noga, Siry 2010].

The biological assessment of the water quality of the Terebowiec stream and its tributary was carried out with use of OMNIDIA software [Lecointe et al. 1993]. For the analysis, the SPI, GDI, TDI and \%PT indices were used, which are the most commonly applied in monitoring studies in Poland [Żelazowski et al. 2004; Dumnicka et al. 2006; Rakowska, Szczepocka 2011; Szczepocka et al. 2014]. The study using diatomaceous indices showed that the Terebowiec stream and its tributary are characterized by good or high ecological status on the basis of the SPI and GDI indices, and the trophic status determined by the TDI index indicates mostly eutophy or mesotrophy. The SPI [Coste in CEMAGREF 1982] and the GDI indices [Coste, Ayphassorho 1991] shows better water quality compared to the TDI index, which determines the degree of the trophic state of water [Kelly, Whitton 1995]. The \%PT values were very low $(1.1-5.2 \%)$ and pointed to the waters being free of organic pollutants. The possibility of 
organic pollutants and the risk of eutrophication is viewed only when the participation is greater than 20\% PT [Kelly, Whitton 1995; Kelly et al. 2001]. The SPI and GDI indices showed similar values in all study seasons, while the TDI index had a worse state of trophy in Autumn. The River San had a better trophic state, on the section under the mouth of the Wołosaty stream into the River San, in the territory of the San Valley Landscape Park [Noga et al. 2014b]. Also, analysis using the statistical method of PCA split position on Terebowiec stream into a two groups (separately spring season and autumn seasons). The Terebowiec tributary is also characterized by lower trophy, and it was distinguished by a separate group in the PCA analysis.

Despite the fact that most of the chemical parameters of the Terebowiec stream indicate very good water quality, diatoms growing in Autumn seasons react to even a slight increase in the fertility of the examined water (nitrate and ammonium ions were always slightly higher in Autumn), which can be related to the increased temperature, lower level of waters and tourist traffic during the summer. It manifests itself mainly via a reduction of the trophic status of studied waters, but the value of the $\% \mathrm{PT}$ index is very low and shows no risks of eutrophication.

\section{Acknowledgements}

Authors wish to thank the Directorate of the Bieszczady National Park for permission to collection of diatom samples.

\section{REFERENCES}

1. Bąk M., Witkowski A., Żelazna-Wieczorek J., Wojtal A.Z., Szczepocka E., Szulc A. \& Szulc B. 2012. Klucz do oznaczania okrzemek w fitobentosie na potrzeby oceny stanu ekologicznego wód powierzchniowych w Polsce. Główny Inspektorat Ochrony Środowiska, Biblioteka Monitoringu Środowiska, Warszawa, 1-452 (in Polish).

2. CEMAGREF 1982. Etude des méthodes biologiques quantitative d'appréciation de la qualité des eaux - Rapport Division Qualité des Eaux Lyon. Agence financiére de Bassin Rhone - Méditerranée - Corse, Pierre-Bénite, 218 pp.

3. Coste M. \& Ayphassorho H. 1991. Étude de la qualité des eaux du Bassin Artois-Picardie a l’aide des communautés de diatomées benthiques (Application des indices diatomiques) - Raport
Cemagref. Bordeaux - Agence de l'Eau ArtoisPicardie, Douai, 277 pp.

4. Czarnecka H. (ed.) 2005. Atlas podziału hydrograficznego Polski. Part 1 and 2. Warszawa (in Polish).

5. Decree of the Minister of the Environment from 22 Oct. 2014 On the classification status of surface waters and environmental quality standards for priority substances (Dz. U. 2014 No 0, pos. 1482).

6. Dumnicka E., Jelonek M., Kwandrans J., Wojtal A. \& Żurek R. 2006. Ichtiofauna i status ekologiczny wód Wisły, Raby, Dunajca i Wisłoki. Institute of Nature Conservation, Polish Academy of Sciences, Kraków, 220 pp.

7. Galas J., Dumnicka E., Galus-Barchan A. 2014. A comparison of various indices based on benthic macrofauna for the assessment of the ecological status of selected Carpathian streams. Oceanological and Hydrobiological Studies 43(2), 123-130.

8. Hofmann G. 1994. Aufwuchs-Diatomeen in Seen und ihre Eignung als Indikatoren der Trophie. Bibliotheca Diatomologica 30, 1-241.

9. Hofmann G., Werum M., Lange-Bertalot H. 2011. Diatomeen im Süßwasser - Benthos von Mitteleuropa. Bestimmungsflora Kieselalgen für die ökologische Praxis. Über 700 der häugfisten Arten und ihre Ökologie. [In:] H. Lange-Bertalot, (Ed.) A.R.G. Gantner Verlag K.G., Ruggell, 908 pp.

10. Kawecka B. 1980. Sessile algae in european mountains streams. 1. The ecological characteristics of communities. Acta Hydrobiol. 22, 361-420.

11. Kawecka B., Sanecki J. 2003. Didymosphenia geminata in running waters of southern Poland Symptoms of change in water quality? Hydrobiol., 495, 193-201.

12. Kelly M.G. \& Whitton B.A. (1995). The Trophic Diatom Index: a new index for monitoring eutrophication in rivers. J. Appl. Phycol. 7, 433-444.

13. Kelly M.G., Adams C., Graves A.C., Jamieson J., Krokowski J., Lycett E.B., Murray-Bligh J., Pritchard S. \& Wilkins C. 2001. The Trophic Diatom Index: A User's Manual. Revised Edition. Environment Agency, Bristol, BS32 4UD, 1-74.

14. Klama H. 2013. Liverworts of the Terebowiec stream valley in the Western Bieszczady Mts. (Polish Eastern Carpathians). Roczniki Bieszczadzkie 21, 42-56 (in Polish with English summary).

15. Klimaszewski M., Starkel L. 1972. Karpaty Polskie. [In:] M. Klimaszewski (ed.): Geomorfologia Polski. I. Polska południowa - góry i wyżyny. Warszawa, PWN, 1-384 pp.

16. Krammer K. 2000. The genus Pinnularia. [In:] H. Lange-Bertalot (ed.): Diatoms of Europe. Vol. 1., 703 pp. A.R.G. Gantner Verlag K.G.

17. Krammer K., Lange-Bertalot H. 1986. Bacillari- 
ophyceae. 1. Naviculaceae. [In:] H. Ettl, J. Gerloff, H. Heyning, D. Mollenhauer (eds): Süsswasserflora von Mitteleuropa 2(1), 876 pp. G. Fischer Verlag, Stuttgart - New York.

18. Krammer K., Lange-Bertalot H. 1988. Bacillariophyceae. 2. Bacillariaceae, Epithemiaceae, Surirellaceae. [In:] H. Ettl, J. Gerloff, H. Heyning, D. Mollenhauer (eds): Süsswasserflora von Mitteleuropa 2(2), 596 pp. G. Fischer Verlag, Stuttgart New York.

19. Krammer K., Lange-Bertalot H. 1991a. Bacillariophyceae. 3. Centrales, Fragilariaceae, Eunotiaceae. [In:] H. Ettl, J. Gerloff, H. Heyning, D. Mollenhauer (eds): Süsswasserflora von Mitteleuropa 2(3), 576 pp. G. Fischer Verlag, Stuttgart - Jena.

20. Krammer K., Lange-Bertalot H. 1991b. Bacillariophyceae. 4. Achnanthaceae, Kritische Ergänzungen zu Navicula (Lineolate) und Gomphonema, Gesamtliteraturverzeichnis. [In:] Ettl, H., Gerloff, J., Heyning, H., Mollenhauer, D. (eds): Süsswasserflora von Mitteleuropa 2(4), G. Fischer Verlag, Stuttgart - Jena, 437 pp.

21. Kukuła K. 2002. Dorzecze Górnego Sanu i jego walory. [In:] F. Oberc (ed.): Zeszyty Archiwum Ziemi Sanockiej. Fundacja Archiwum Ziemi Sanockiej, Sanok-Zahutyń, 2, 30-35.

22. Kukuła K., Szczęsny B. 2000. Ekologiczne uwarunkowania ochrony ekosystemów wodnych Bieszczadów Zachodnich. Monografie Bieszczadzkie 10, 79-114.

23. Lange-Bertalot H. 2001. Navicula sensu stricto. 10 genera separated from Navicula sensu lato. Frustulia. [In:] H. Lange-Bertalot, (ed.): Diatoms of Europe. Vol. 2., 526 pp., A.R.G. Gantner Verlag K.G.

24. Lecointe C., Coste M. \& Prygiel J. 1993. OMNI DIA, a software for taxonomy, calculation of diatom indices and inventory management. Hydrobiologia 269/270: http://dx.doi.org/509-513. http:// dx.doi.org/10.1007/BF00028048

25. Levkov Z. 2009. Amphora sensu lato. [In:] H. Lange-Bertalot (ed.) Diatoms of Europe. Koeltz Scientific Books, Köningstein/Germany, 916 pp.

26. Noga T. (2012). Diversity of diatom communities in the Wisłok River (SE Poland). In: K. Wołowski, I. Kaczmarska, J.M. Ehrman \& A.Z. Wojtal, (eds): Phycological Reports: Current advances in algal taxonomy and its applications: phylogenetic, ecological and applied perspective. (pp. 109-128). Institute of Botany Polish Academy of Sciences, Kraków.

27. Noga T. \& Siry K. (2010). Diversity of diatom flora in the Łubienka stream (The Dynów Foothills, south-eastern Poland). Zeszyty naukowe PTIE i PTG 12: 75-86, (in Polish with English summary).

28. Noga T., Stanek-Tarkowska J., Peszek Ł., Pajączek A., Kowalska S. 2013a. Use of dia- toms to assess water quality of anthropogenically modified Matysówka stream. Journal of Ecological Engineering 14(2): 1-11. http://dx.doi. org/10.5604/2081139X.1043166

29. Noga T., Stanek-Tarkowska J., Pajączek A., Peszek Ł., Kochman N., Woźniak K. 2013b. Application of diatoms to assess the quality of the waters of the Baryczka stream, left-side tributary of the River San. Journal of Ecological Engineering 14(2), 8-23. http://dx.doi.org/10.5604/2081139X.1055818

30. Noga T., Kochman N., Peszek Ł., Stanek-Tarkowska J., Pajączek A. 2014a. Diatoms (Bacillariophyceae) in rivers and streams and on cultivated soils of the Podkarpacie Region in the years 2007-2011. Journal of Ecological Engineering 15(1), 6-25. http://dx.doi.org/10.12911/22998993.1084168

31. Noga T., Stanek-Tarkowska, Pajączek A., Kochman N., Peszek Ł. 2014b. Ecological assessment of the San River water quality on the area of the San Valley Landscape Park. Journal of Ecological Engineering 15(4), 12-22. http://dx.doi. org/10.12911/22998993.1125453

32. Pajączek A., Musiałek M., Pelczar J., Noga T. 2012. Diversity of diatoms Bacillariophyceae) in the Mleczka River, Morwawa River and Rożanka stream (tributaries of the Wisłok River, SE Poland), with particular reference to threatened species. [In:] K. Wołowski, I. Kaczmarska, J.M. Ehrman, A.Z. Wojtal (Eds), Current advances in algal taxonomy and its applications: phylogenetic, ecological and applied perspective. W. Szafer Institute of Botany, Polish Academy of Sciences, Kraków, 129-151.

33. Peszek Ł., Noga T., Stanek-Tarkowska J., Pajączek A., Kochman-Kędziora N., Pieniążek M. 2015. The effect of anthropogenic change in the structure of diatoms and water quality of the Żołynianka and Jagielnia streams. Journal of Ecological Engineering 16(2), 33-51. http://dx.doi. org/10.12911/22998993/1856

34. Potapowa M. 2006. Achnanthidium zhakovschikovii sp. nov. (Bacillariophyta) and related species from rivers of Northwestern Russia. Nova Hedwigia 82(3-4), 399-408.

35. Przybylska K., Kucharzyk S. 1999. Skład gatunkowy i struktura lasów Bieszczadzkiego Parku Narodowego. Monografie Bieszczadzkie 6, 1-159 (in Polish).

36. Rakowska B., Szczepocka E. 2011. Demonstration of the Bzura River restoration using diatom indices. Biologia 66(3), 411-417.

37. Siemińska J., Bąk M., Dziedzic J., Gąbka M., Gregorowicz P., Mrozińska T., Pełechaty M., Owsiany P.M., Pliński M., Witkowski A. 2006. Red list of the algae in Poland - Czerwona lista glonów w Polsce. [In:] Z. Mirek et al. (Eds) Red list of 
plants and fungi in Poland - Czerwona lista roślin i grzybów Polski. Polish Academy of Sciences, Kraków, 35-52.

38. Szczepocka E., Szulc K., Szulc B., Rakowska B., Żelazna-Wieczorek J. 2014. Diatom indices in the biological assessment of the water quality based on the example of a small lowland river. Oceanological and Hydrobiological Studies 43(3), 265-273. http://dx.doi.org/10.2478/s13545-014-0141-z

39. Ter Braak C.J.F., Šmilauer P. 2012. Canoco reference manual and user's guide. Software for ordination (version 5.0). Microcomputer Power, Ithaca, NY, USA. 496 pp.

40. Winnicki T., Zemanek B. 2009. Nature in the Bieszczady National Park. Published by the Bieszczady National Park, Ustrzyki Dolne, 1-178.

41. Zemanek B. 1991. The phytogeographical division of the Polish East Carpathians. Zeszyty Nauk.
Uniw. Jagiell., Prace Bot. 22, 81-119.

42. Wołowski K. 2011. Badania wstępne nad eugleninami i innymi glonami torfowiska Wołosate w Bieszczadzkim Parku Narodowym. Fragm. Flor. Geobot. Polonica 18(1), 131-146.

43. Żarnowiec J. 2010. Mosses of the Terebowiec stream valley (Western Bieszczady Mts, Bieszczady National Park). Roczniki Bieszczadzkie 18, 157-166 (in Polish with English summary).

44. Żelazna-Wieczorek J. 2012. Okrzemki Bacillariophyta źródeł i odcinków źródłowych potoków w górnym odcinku rzeki San. Roczniki Bieszczadzkie 20, 220-229.

45. Żelazowski E., Magiera M., Kawecka B., Kwandrans J. \& Kotowicz J. 2004. Use of algae for monitoring rivers in Poland - in the light of a new law for environmental protection. Oceanological and Hydrobiological Studies 33(4), 27-39. 